\title{
The Relationship Between Early Sexual Debut and Psychosocial Outcomes: A Longitudinal Study of Dutch Adolescents
}

\author{
Wadiya Udell $\cdot$ Theo Sandfort $\cdot$ Ellen Reitz $•$ \\ Henny Bos $\cdot$ Maja Dekovic
}

Received: 7 August 2008/Revised: 13 November 2009/ Accepted: 13 November 2009/Published online: 30 January 2010

(C) The Author(s) 2010. This article is published with open access at Springerlink.com

\begin{abstract}
In a longitudinal dataset of 470 Dutch adolescents, the current study examined the ways in which early sexual initiation was related to subsequent attachment, self-perception, internalizing problems, and externalizing problems. For male adolescents, analyses revealed general attachment to mother and externalizing problems at Wave 1 to predict to early transition at Wave 2. However, there was no differential change in these psychosocial factors over time for early initiators of sexual intercourse and their non-initiating peers. For female adolescents, the model including psychosocial factors at Wave 1 did not predict to sexual initiation at Wave 2. However, univariate repeated measures analyses revealed early initiators to have significantly larger increases in self-concept and externalizing problems than their non-initiating female peers. While the
\end{abstract}

\footnotetext{
W. Udell ( $\square)$

Department of Interdisciplinary Arts and Sciences, University of Washington Bothell, Box 358511, 18115 Campus Way NE, Bothell, WA 98011, USA

e-mail:wau2@u.washington.edu

T. Sandfort

HIV Center for Clinical and Behavioral Studies, New York State Psychiatric Institute and Columbia University, New York, NY, USA

E. Reitz · M. Dekovic

Faculty of Social Sciences in the Research Centre Psychosocial Development in Context, Utrecht University, Utrecht,

The Netherlands

H. Bos

Department of Education, Faculty of Social and Behavioral Sciences, University of Amsterdam, Amsterdam, The Netherlands
}

difference between female early initiators and non-initiators were statistically significant, the mean levels of problem behaviors were very low. The findings suggest that, contrary to previous research, early sexual initiation does not seem to be clustered with problem behaviors for this sample of Dutch adolescents.

Keywords Early sexual debut · Adolescence · Longitudinal $\cdot$ Psychosocial $\cdot$ Attachment $\cdot$ Self-concepts

\section{Introduction}

Early initiation of sexual intercourse has long been an area of interest to developmental psychologists, health, and sex researchers. Evidence suggests the timing of sexual intercourse and sequence of sexual trajectories (e.g., pace and sequence at which adolescents progress from less intimate to more intimate behaviors) are related to health outcomes (de Graaf, Vanwesenbeeck, Meijer, Woertman, \& Meeus, 2009). Research focusing on U.S. samples has found evidence of a relationship between sexual intercourse during early adolescence (commonly defined as before age 16) and future problem behaviors (Cavazos-Rehg et al., 2009; Woodward, Fergusson, \& Horwood, 2001). Adolescents who have initiated sexual intercourse during early adolescence were more likely to have used drugs, alcohol, and engaged in delinquent activities compared to those who have not engaged in sexual intercourse at early ages (Ketterlinus, Lamb, \& Nitz, 1994; Paul, Fitzjohn, Herbison, \& Dickson, 2000). Research has also found early sexual intercourse to share some of the same psychosocial predictors (e.g., self-efficacy, self-concept, perceived risk, peer norms, relationships with parents and peers, etc.) with various problem behaviors (Biglan et al., 1990; Black, Ricardo, \& Stanton, 1997; Miller et al., 1997). Adolescents who have poor quality relationships with their 
mothers are more likely to initiate sexual activity at early ages (Davis \& Friel, 2001), engage in delinquent activities, and use alcohol and other substances compared to youth with close attachments to their mothers (Sokol-Katz, Dunham, \& Zimmerman, 1997; Webb, Baer, McLaughlin, McKelvey, \& Caid, 1991).

Sexual intercourse during early adolescence has been mainly viewed as a problem behavior (Jessor \& Jessor, 1977; Ream, 2006) alongside behaviors such as drug use, running away, stealing, vandalism, and school failure or drop out. However, researchers have questioned this unidimensional approach to understanding the timing of sexual intercourse during adolescence (Ehrhardt, 1996; Sandfort, Orr, Hirsh, \& Santelli, 2008; Savin-Williams \& Diamond, 2004). Various researchers have argued that although early sexual intercourse is potentially harmful due to its association with future sexual risk behaviors (Greenberg, Magder, \& Aral, 1992; Smith, 1997), there are important differences between early intercourse and the problem behaviors to which it is linked. Unlike drug use and other forms of delinquent behavior, engaging in sexual intercourse is a normative aspect of development that becomes increasingly more normative as individuals mature into adulthood (Sandfort et al., 2008). Given the increasingly normative aspect of sexual intercourse during late adolescence and emerging adulthood, early sexual intercourse in itself may not serve as a catalyst for problem behaviors and maladjustment for youth.

Although research has illustrated an association between early sexual intercourse and negative psychosocial factors (Kirby, Lepore, \& Ryan, 2005), the majority of this work has been based on cross-sectional comparisons (Biglan et al., 1990; Small \& Luster, 1994), making it difficult to determine whether the associated negative psychosocial factors preceded or followed initiation of intercourse. Recent work by Armour and Haynie (2007) utilized a longitudinal design to examine the direction of the relationship between early intercourse and delinquency. They found early sexual intercourse to be associated with delinquency one year later (Armour \& Haynie, 2007). While this study is a step forward in understanding the nature of the relationship between intercourse during early adolescence and negative outcomes, the study was limited in its examination of only one psychosocial factor. Other longitudinal studies utilizing psychosocial data to predict early sexual debut have also been conducted (Laflin, Wang, \& Barry, 2008; Santelli et al., 2004), allowing further insight into the order of the relationship between early intercourse and psychosocial factors. However, many of these studies only examined factors that preceded initiation of sexual intercourse, and not whether and/or how such factors change following first intercourse. While using longitudinal designs to examine predictors of sexual initiation is informative, it leaves open the question of whether and how such factors change following the debut of sexual intercourse. Given the current research available, longitudinal research focusing on both the period before and after first intercourse is needed to gain a better understanding of the relationship between early initiation of sexual intercourse and subsequent psychosocial outcomes.

In addition to considering the effect of timing of sexual intercourse on future psychosocial development, it is important to consider the potential influence of sociocultural factors on initiation of sexual intercourse. Sociocultural and ecological theorists caution against assumptions of universal developmental mechanisms, and argue for the need to study individuals and developmental processes in context (Bronfenbrenner, 1979; Rogoff, 2003). Although many researchers focus on contexts more remote to the adolescent (e.g., family, peers, school), more distal contexts also influence human development. Macrosystemic factors, like social cultures (e.g., national, religious, or cultural beliefs and or practices), may also influence the relationship between early intercourse and psychosocial development (Krieger, 2001). It is, therefore, important to consider broader cultural contexts, such as societal beliefs regarding sexuality when studying the relationship between early initiation and psychosocial development. Given the differing beliefs concerning both the period of adolescence and the meanings of various sexual practices across cultures, it is possible that examining the relationship between early sexual intercourse and psychosocial outcomes for adolescents from different cultural backgrounds may lead to results different from that of studies based on U.S. samples. It is, therefore, important to extend studies of the relationship between early intercourse and psychosocial factors associated with adolescent development to include examinations of youth from various cultures. The present study addressed this important area by focusing on a sample of Dutch adolescents. Additionally, this study extended previous research by examining: (1) psychosocial predictors of early sexual initiation and (2) whether and how these factors change following early initiation.

Psychosocial Factors Associated with Adolescent Problem Behaviors

\section{Attachment}

Although a moderate level of conflict characterizes parentadolescent relationships, parental attachment remains an important factor for adolescent psychosocial adjustment as closeness between parents and adolescents remains stable over time (Smetana, Campione-Barr, \& Metzger, 2006; Steinberg, \& Morris, 2001). Insecure maternal attachment or poor quality relationships with mothers has been associated with risky sexual behavior and other problem behaviors (Cooper, Shaver, \& Collins, 1998). In comparison to research on maternal attachment, findings on the relationship between paternal attachment and adolescent problem behaviors have been less robust. Some studies have found paternal attachment to be related to various adolescent psychosocial outcomes (e.g., depression, 
externalizing behaviors, and peer relations) (Lieberman, Doyle, \& Markiewicz, 1999; Liu, 2006; Williams \& Kelly, 2005), whereas other studies have found minimal or no relationship between adolescent adjustment and paternal attachment (Arbona $\&$ Power, 2003). Based on previous research, we expected Dutch adolescents who have low levels of attachment to their mothers to be more likely to initiate sexual intercourse earlier than their peers who report high levels of maternal attachment. We have no hypotheses regarding changes in attachment following transition to sexual intercourse. Likewise, we made no predictions regarding the relationship between initiation of sexual intercourse and paternal attachment.

Attachment to peers becomes increasingly important during adolescence (Smetana et al., 2006), and some have argued that peer attachment figures become more influential than parent attachment figures during this time (Cooper et al., 1998). While parents have been found to influence adolescents' values and moral issues, adolescents' social behaviors are more influenced by their peers (Smetana et al., 2006). Peer attachment has been found to strengthen the effects of peer beliefs about sex on adolescent sexual behavior (Manning, Longmore, \& Giordano, 2005). Previous research does not allow for predictions on the relationship between quality of peer attachment and initiation of sexual intercourse, as initiators and non-initiators could have either strong or weak attachment to peers who may or may not endorse sexual intercourse for adolescents.

\section{Self-Concept}

Adolescents evaluate themselves along several distinct dimensions, and the emergent self-concepts and self-worth serve as important factors for positive youth development (Masten et al., 1995; Steinberg \& Morris, 2001). Studies have found mixed relationships between self-concepts (and competencies) and adolescent adjustment (Masten et al., 1995; Vermeiren, Bogaerts, Ruchkin, Deboutte, \& Schwab-Stone, 2004). For example, Williams, Connolly, and Cribbie (2008) found peer self-concept (i.e., perceptions of one's peer relationships) to be positively related to engagement in sexual intercourse among a population of 12 and 13 year old adolescents. Similarly, Houlihan et al. (2008) found initiation of sexual intercourse to be associated with an increase in self-concept among a population of African American youth. However, many other studies have found positive self-concepts to be related to safer sexual behaviors (Salazar et al., 2004, 2005). Additionally, adolescents with low self-worth have been found to engage in more risky sexual behavior than adolescents with higher self-worth (Crockett, Moilanen, Raffaelli, \& Randall, 2006). Given the inconsistencies in previous research on the relationship between self-concept and sexual risk behavior, we make no predictions regarding the relationship between self-concept and initiation status for this sample of Dutch adolescents.

\section{Internalizing and Externalizing Problems}

The negative impact of internalizing problems (e.g., depression) and externalizing problems (e.g., aggression, substance abuse, delinquency, etc.) on positive youth development is well known (Alloy, Zhu, \& Abramson, 2003; Flannery, Vazsonyi, \& Rowe, 1996; Lerner \& Galambos, 1998; Winters, August, \& Leitten, 2003). In regards to its relationship with sexual behavior, male and female adolescents with externalizing problems have been found to be more likely to initiate sexual intercourse, and engage in riskier sexual behaviors than their non-externalizing peers (Caminis, Henrich, Ruchkin, Schwab-Stone, \& Martin, 2007; Donenberg, Bryant, Emerson, Wilson, \& Pasch, 2003). In contrast, research on internalizing problems has been more inconsistent. Whereas some studies have found male and female adolescents with internalizing problems to be less likely to initiate sexual intercourse than their non-internalizing peers (Caminis et al., 2007), others have found a positive association between internalizing problems and sexual initiation for both male and female adolescents (Longmore, Manning, Giordano, \& Rudolf, 2004). Based on previous findings, we expected externalizing problems to predict early initiation, with adolescents with higher levels of externalizing problems being more likely to be early initiators. We have no hypotheses regarding how externalizing problems may change following early initiation. Given the conflicting findings regarding internalizing problems, we make no prediction regarding the relationship between internalizing problems and initiator status.

The current study examined the relationship between sexual initiation during early adolescence and psychosocial factors related to adolescent development among a population of Dutch adolescents. Focusing on a Dutch population is important, given the few studies examining predictors and the effects of early sexual initiation in this population. Based on what is known from previous research of non-Dutch samples on the relationship between psychosocial factors and timing of coital debut, this exploratory study addressed the following questions: (1) Are Dutch adolescents who have weaker attachment and self-concepts, and more internalizing problems and externalizing problems, more likely to initiate sexual intercourse earlier than their peers? and (2) Are there differential changes in attachment, self-concept, and internalizing and externalizing problems over time for Dutch adolescents who have engaged in early sexual intercourse compared to those who have not?

Given widely documented gendered double standard regarding sexuality and sexual initiation (Ravesloot, 1997; Worthington, Savoy, Dillon, \& Vernaglia, 2002), analyses were run separately for male and female adolescents. Researchers have long argued that whereas females are taught not to behave as sexual beings, societal male gender role expectations dictate and reinforce the notion that males should engage in various forms of heterosexual sexual contact at early ages and with regular frequency (Martin, 1996; Stevens, 2001). Given differing 
gendered expectations regarding sexuality, it is likely that the relationship between early sexual intercourse and the psychosocial factors examined in this study will be different for male and female adolescents.

\section{Method}

Participants and Procedure

This study was part of a larger longitudinal study examining the development of problem behaviors among a sample of 650 Dutch adolescents (Reitz, Dekovic, \& Meijer, 2006). Participants were drawn from eighth grade classrooms from three secondary schools in the Netherlands, located in medium- to large-sized municipalities. Ten schools were approached to participate. However, three of these schools were unwilling to participate. After schools agreed to participate, passive informed consent, where parents had the opportunity to decline participation, was obtained from the parents. A letter containing information about the date and nature of the study were handed out to all adolescents to bring home to their parents. The letter explained to both parents and adolescents that we were interested in examining the types of interactions adolescents have with their parents and friends, as well as the type of problem behaviors that may occur during puberty. The letter also explained that we were given permission from their child's school to conduct the study, and that they were able to decline participation by indicating so on the response form. Parents could send the letter back, indicating that they declined to allow their child to participate in the study. Less than $1 \%$ of the adolescents in each of the target schools had their participation withheld by parents. Adolescents were not compensated for participation. Approval of this study was granted by the participating schools. Approval by Internal Review Boards are not required in the Netherlands for this type of research.

Participants were tested twice, with a year interval in between the visits. Students were given a battery of questionnaires in their classrooms during regular school hours at both Wave 1 and Wave 2. Participants completed the battery at their individual desks. The desk arrangements provided a level of privacy, consistent with regular test-taking procedures at secondary schools. A packet, including the assessment battery and a self-addressed postage paid envelope, was sent to the homes of students who were absent during the data collection. Nonresponders were called at home to ask whether they would complete the questionnaire and send it back. Participant attrition from Wave 1 to Wave 2 was $13.4 \%$.

For the purpose of this study, only those adolescents who indicated they had not initiated sexual intercourse at Wave 1 were selected for the analyses $(N=470)$. Participants were between the ages of 12 and 15 years $(M=13.31, S D=.51)$. Of the 470 adolescents included in the analyses, $258(55 \%)$ were females and 212 (45\%) were males. A total of 430 (92\%) adolescents were Dutch nationals and $40(8 \%)$ adolescents were non-nationals (from Morocco, Turkey, Surinam, Dutch Antilles, and other countries not identified). Percentage of Dutch nationals and non-nationals were roughly equal for males $(92 \%$ Dutch nationals) and females (91\% Dutch nationals). Of the 470 adolescents, $67 \%(N=317)$ attended academic schools, and $33 \%(N=153)$ attended vocational schools. The percentage of male and female adolescents attending vocational and academic schools were similar. Thirty-one percent of males $(N=66)$ and $34 \%$ of females $(N=87)$ attended a vocational school. Sixtynine percent of males $(N=146)$ and $66 \%$ of females $(N=171)$ attended an academic school. Fourteen percent $(N=65)$ of the sample indicated they had sexual intercourse at Wave 2. We refer to these youth as transitioners. The sample of transitioners consisted of slightly more females $(N=39$ or $60 \%)$ than males $(N=26$ or $40 \%)$. The percentages of male transitioners were similar across the age groups. Transitioners accounted for $13 \%$ of the 13 year olds, and $10 \%$ of the 14 year-olds. There were no transitioners among the 12 and 15 year olds males. Given the limited number of 12 year-old $(N=1)$ and 15 year-old $(N=3)$ males, percentages of transitioners and non-transitioners were roughly the same for these two age groups. The percentages of female transitioners also did not vary largely between the three age groups. Transitioners accounted for $13 \%$ of 13 year olds, $20 \%$ of 14 year olds, and $25 \%$ of 15 year-olds. There were no 12 year-old females in the sample.

\section{Measures}

\section{Sexual Intercourse}

Initiation of sexual intercourse was measured by one item that asked participants whether they "had ever slept with somebody" (translated into English).

\section{Perceived Quality of Relationship to Parents and Peers}

A short version of the Inventory of Parent and Peer Attachment (IPPA; Armsden \& Greenberg, 1987; Raja, McGee, \& Stanton, 1992) was used to determine the quality of affectional bonds between adolescents and their mothers, fathers, and peers. Separate measures assessed relationship to mother, father, and to peers. Each scale (mother, father, and peer versions) consisted of 12 items and contained three subscales: Communication, Trust, and Alienation. Each subscale consisted of four items. The Communication subscale measured the extent to which adolescents experienced high quality communication with their mother, father, and peers (e.g.. "If my mother knows something is bothering me, she asks me"). The Trust subscale measures the extent to which adolescents trust their mother, father, and peers to accept and respect their feelings and wishes (e.g., "When I talk 
to my father about my problems, I feel embarrassed or stupid"). Finally, the Alienation scale measured the extent to which adolescents experience negative feelings toward and feelings of disconnection from their mother, father, and peers (e.g., "I wish I had other friends"). Items were rated on a 4-point Likert scale. Subscale scores were averaged across items resulting in a mean score with a range of $1-4$. Overall attachment was a composite of the three subscales. Higher scores represent closer attachment. Alphas for overall attachment for Wave 1/Wave 2 were $.82 / .82, .80 / .78$, and $.86 / .83$ for mother, father, and peers, respectively.

\section{Self-Concept}

The 35-item Self-Perception Profile for Adolescents (SPPA; Harter, 1988) was used to measure adolescents' evaluation of their competence in the following domains: scholastic competence, social acceptance, athletic competence, physical appear ance, romantic appeal, close friendship, and global self-worth. For each item, two contrasting descriptions were presented with two options for each description. Participants were first asked to decide which description best described them, the description on the left or the right (e.g., close friendship: some youth have a lot of friends (left side); other youth do not have a lot of friends (right side)). When the decision was made, the second step was to decide whether the description on the selected side was "sort of true" or "really true" for them. Participants therefore had a choice of one of four possible options for each item ("sort of true" or "really true" from the statement on the right hand side or from the statement on the left hand side). Each item was scored from 1 to 4. Scale scores for each domain were averaged across items resulting in a mean score with a range of $1-4$. Higher scores indicated greater self-perceived competence in the respective domains. Internal consistencies for Wave 1/Wave 2 were $.59 / .60$ for scholastic competence, .73/.79 for social acceptance, $.85 / .86$ for athletic competence, $.82 / .83$ for physical appearance, .67/.70 for romantic appeal, .65/.61 for close friendship, and .78/.80 for global self-worth.

\section{Problem Behavior: Externalizing and Internalizing}

A subset of the Youth Self-Report (YSR; Achenbach, 1991; Verhulst, Van der Ende, \& Koot, 1997) was used to obtain adolescent reports regarding their own externalizing and internalizing syndromes. A total of 62 items measuring rule-breaking behavior (referred to as delinquent behavior in older scales), aggression, anxiety/depression, withdrawal, and somatic complaints were included in the survey. All items were rated on a 3-point Likert scale where 0 indicates responses of "not true," 1 "somewhat true," and 2 "very true or often true." Scores for each syndrome was averaged across items resulting in a mean score for each subscale with a range of $0-2$.
Measures of externalizing syndromes or problems consisted of 11 items measuring rule-breaking behavior (e.g., "I steal from home") and 19 items assessing aggressive behavior (e.g., "I fight a lot"). Alphas for the rule-breaking and aggressive behavior for Wave 1/Wave 2 in this study were $.67 / .69$ and $.82 / .82$, respectively.

In order to expand the range of externalizing problems to include those frequently occurring among non-clinical groups within the targeted age group, two additional measures of school problems and disobedience were developed (Reitz, Dekovic, \& Meijer, 2005). Assessment of school problems consisted of seven items adapted from several measures (e.g., Brack, Brack, \& Orr, 1994; Gillmore et al., 1991). Items assessed a variety of school-based misconduct, such as whether adolescents have been sent out of class for misbehavior (Gillmore et al., 1991) and whether they have copied homework from others (Fletcher, Steinberg, \& Sellers, 1999). Assessment of disobedience consisted of eight items adapted from several measures (e.g., Maggs, Almeida, \& Galambos, 1995; Rothbaum \& Weisz, 1994). Items assessed various forms of disobedience to parents, such as whether adolescents refused to do domestic chores (Peeters, 1994), and whether they ignored prohibitions from parents (Dekovic, 1999). Both assessments were created to conform to the YSR format. Items were rated on a 3-point Likert scale, with a range of $0-2$ for each subscale. Alphas for Wave 1/Wave 2 were .65/.74 for school problems and .71/.74 for disobedience.

Measures of internalizing problems consisted of 16 items measuring Anxiety/Depression (e.g., "I feel worthless or inferior"), 7 items measuring feelings of Withdrawal (e.g., "I rather be alone than with others"), and 9 items measuring Somatic Complaints (e.g., "I feel overtired"). Items were rated on a 3-point Likert scale, with a range of $0-2$ for each subscale. Alphas for the Anxiety/Depression, Withdrawal, and Somatic Complaints for Wave 1/Wave 2 in this study were .84/.87, .65/ .68 , and $.73 / .76$, respectively.

Participant ethnicity was assessed by asking respectively where the participant and his/her mother and father were born. If participants indicated any of the individuals were born outside of the Netherlands, they were categorized as non-Dutch. This categorization of ethnicity is consistent with Dutch demographic practices.

\section{Statistical Analysis}

All analyses were conducted separately for males and females. Data analysis for both males and females were conducted in three phases. Imputed values for missing dependent variable items were calculated separately for males and females using mean substitution at the level of participants.

To examine predictors of early sexual initiation at Wave 2, phase one consisted of a logistic regression analysis where 
transitioner was coded as 0 and non-transitioner was coded as 1. The logistic regression controlled for potential differences due to ethnicity (Dutch vs. Other) and type of education/ school (vocational/trade vs. academic) — two variables found related to psychosocial outcomes in previous studies of Dutch adolescents (Dekovic, Pels, \& Model, 2006; Swertz, Duimelaar, Thijssen, Harmsen, \& Vergeer, 2003). In the first model, initiation status was regressed on ethnicity and type of education. The second model included ethnicity and type of education in the first block, and the psychosocial variables (i.e., attachment, self-concept, internalizing and externalizing variables) at Wave 1 in the second block.

Given our focus on a population not largely represented in research on early sexual initiation, along with our limited understanding of how early initiation may influence subsequent psychosocial factors, it was important to conduct an in-depth examination of this relationship in order not to miss potential differences between transitioners and non-transitioners. Therefore, phases two and three consisted of two types of analyses to examine changes in psychosocial factors over time. In the second phase, a 2 (transition status) by 2 (time) multivariate analysis of covariance (MANCOVA) was conducted, controlling for differences due to ethnicity and type of education. The examination of whether there was an overall significant time by sexual status interaction in the dependent variables was conducted at $p=.05$. When the MANCOVA revealed an interaction, post hoc univariate results were examined to determine which variables independently contributed to the overall significance (Stevens, 1992).

In the third phase of analyses, we conducted a series of analyses whereby each of the attachment, self-concept, internalizing, and externalizing variables was individually analyzed using a 2 (transition status) $\times 2$ (time) repeated measures analysis of covariance (ANCOVA), where ethnicity and type of education were entered as covariates. The analyses compared transitioners to non-transitioners over time (from Wave 1 to Wave 2) on the variables of interest.

\section{Results}

Psychosocial Predictors of Transition Status for Males

Results from the first model including the covariate variables of ethnicity and type of education indicated the model to be statistically significant, $\chi^{2}=9.19, p=.01, d f=2$ (see Table 1). Examination of the individual variables in the first model revealed a significant relationship between type of school and sexual initiation status for males $(p=.003)$. In the second model, the covariates were entered in the first block, and the attachment, self-concept, externalizing, and internalizing variables were entered into the second block. Results indicate this overall model to be statistically significant, $\chi^{2}=41.80$, $p=.002, d f=19$. Additionally, the second block including the psychosocial variables was also significant, $\chi^{2}=32.61$, $p=.01, d f=17$, indicating that after removing variance associated with type of school, the combination of psychosocial factors significantly predicted sexual initiation status. Of the 17 psychosocial variables, maternal attachment ( $p=$ $.05)$, rule-breaking behavior $(p=.01)$, and aggressive behavior $(p=.04)$ significantly predicted sexual initiation for males. Specifically, male adolescents who indicated more maternal attachment were more likely to be non-transitioners by Wave $2(\mathrm{OR}=1.57)$. Additionally, males who reported more rulebreaking and aggressive behaviors were less likely to be nontransitioners by Wave $2(\mathrm{OR}=.02$ and $\mathrm{OR}=.09$ for rulebreaking and aggressive behaviors, respectively).

\section{Differences Between Male Transitioners} and Non-Transitioners Over Time

A 2 (transition status) $\times 2$ (time) MANCOVA revealed no differences between transitioner and non-transitioner male adolescents over time, $F(17,402)<1$ (see Table 2 for variable means).

\section{Differential Change in Male Transitioners and Non-Transitioners Over Time}

A series of analyses were conducted whereby each psychosocial variable was individually analyzed using a 2 (transition status) $\times 2$ (time) repeated measures ANCOVA, where ethnicity and school-type were entered as covariates. There were no significant time by transition status interactions found for any of the psychosocial variables examined among male adolescents.

\section{Psychosocial Predictors of Transition Status for Females}

Results from the first model including the covariate variables of ethnicity and type of education indicated the model to be statistically significant, $\chi^{2}=10.81, p=.004, d f=2$ (see Table 1). Examination of the individual variables in the first model revealed a significant relationship between sexual initiation status and type of school for females $(p=.007)$. In the second model, the covariates were entered into the first block, and the attachment, self-concept, externalizing, and internalizing variables were entered into the second block. Results indicated the second model including both the covariates and the psychosocial predictors to be statistically significant, $\chi^{2}=32.39, p=.02, d f=19$. However, analysis of the second block in this model indicated that after removing variance associated with ethnicity and type of school, the combination of psychosocial factors did not significantly predict sexual initiation status, $\chi^{2}=21.57, p=.20, d f=17$. As Table 1 shows, the only psychosocial variable in the second block that was a significant predictor of sexual 
Table 1 Odds ratios (OR) and 95\% confidence intervals (CI) for predicting initiation of sexual intercourse by Wave 2, using psychosocial variables measured at Wave 1, controlling for ethnicity and type of school

\begin{tabular}{|c|c|c|c|c|c|c|c|c|}
\hline & \multicolumn{2}{|c|}{ Model 1 males $(n=212)$} & \multicolumn{2}{|c|}{ Model 2 males $(n=212)$} & \multicolumn{2}{|c|}{ Model 1 females $(n=258)$} & \multicolumn{2}{|c|}{ Model 2 females $(n=258)$} \\
\hline & OR & $\mathrm{CI}$ & OR & CI & OR & $\mathrm{CI}$ & OR & $\mathrm{CI}$ \\
\hline Ethnicity & 1.07 & {$[.75,1.54]$} & 1.04 & {$[.68,1.57]$} & 1.66 & {$[.88,3.13]$} & 1.96 & {$[.93,4.12]$} \\
\hline Type of schooling & $3.62 * * *$ & {$[1.55,8.41]$} & $6.13 * * *$ & {$[2.03,18.47]$} & $2.61 * *$ & {$[1.29,5.25]$} & $2.24 *$ & {$[1.04,4.84]$} \\
\hline Attachment (mother) & & & $1.57 *$ & {$[1.00,2.49]$} & & & 1.46 & {$[.95,2.24]$} \\
\hline Attachment (father) & & & 1.25 & {$[.78,2.00]$} & & & .92 & {$[.65,1.31]$} \\
\hline Attachment (peers) & & & .61 & {$[.37,1.03]$} & & & 1.13 & {$[.78,1.63]$} \\
\hline Scholastic competence & & & 1.17 & {$[.21,6.38]$} & & & 1.46 & {$[.43,4.91]$} \\
\hline Social acceptance & & & 1.77 & {$[.20,15.48]$} & & & 2.23 & {$[.53,9.23]$} \\
\hline Athletic competence & & & .29 & {$[.03,2.32]$} & & & .98 & {$[.19,4.88]$} \\
\hline Physical appearance & & & .94 & {$[.12,7.02]$} & & & .99 & {$[.20,4.90]$} \\
\hline Romantic appeal & & & .36 & {$[.04,3.02]$} & & & .36 & {$[.09,1.38]$} \\
\hline Close friendship & & & 1.24 & {$[.23,6.45]$} & & & .72 & {$[.23,2.26]$} \\
\hline Global self-worth & & & .48 & {$[.06,3.63]$} & & & 1.89 & {$[.49,7.19]$} \\
\hline Rule-breaking & & & $.02 *$ & {$[.00, .50]$} & & & $.05^{*}$ & {$[.00, .74]$} \\
\hline Aggression & & & $.09 *$ & {$[.01, .93]$} & & & 2.08 & {$[.17,24.69]$} \\
\hline School problems & & & .54 & {$[.03,8.24]$} & & & .60 & {$[.05,6.41]$} \\
\hline Disobedience & & & 2.03 & {$[.27,15.40]$} & & & 2.56 & {$[.45,14.52]$} \\
\hline Anxiety/depression & & & .64 & {$[.03,12.97]$} & & & 2.10 & {$[.30,14.55]$} \\
\hline Withdrawal & & & 8.16 & {$[.57,115.83]$} & & & .81 & {$[.16,4.01]$} \\
\hline Somatic complaints & & & 2.75 & {$[.28,26.87]$} & & & .38 & {$[.11,1.30]$} \\
\hline
\end{tabular}

$* * * p<.005, * * p<.01, * p<.05$

initiation for females was rule-breaking $(p=.02)$, with female adolescents reporting more rule-breaking being more likely to be transitioners by Wave 2 .

Differences Between Female Transitioners and Non-Transitioners Over Time

A 2 (transition status) $\times 2$ (time) MANCOVA revealed no significant differences between transitioner and non-transitioner female adolescents over time, $F(17,494)=1.49, p=$ .09 (see Table 1 for variable means).

Differential Change in Female Transitioners and Non-Transitioners Over Time

A series of analyses were conducted, whereby each psychosocial variable was individually analyzed using a 2 (transition status) $\times 2$ (time) repeated measures ANCOVA, where ethnicity and school-type were entered as covariates. A significant time by transition status interaction was found for one selfconcept variable and three externalizing variables: perceptions of school skills, disobedience to parents, school problems, and aggressive behavior.

As shown in Table 2, non-transitioners' perceptions of their scholastic competence barely increased over time, whereas transitioners' perceptions increased over the one-year period, $F(1,254)=7.12, p=.008$. Similarly, disobedience to parents increased over time for transitioners, but stayed the same for non-transitioners, $F(1,254)=9.56, p=.002$. Although an increase in school problems was found for both transitioners and non-transitioners, the means in Table 2 show a significantly higher increase in school problems by transitioners, $F(1$, $254)=5.24, p=.02$. Analyses also revealed an increase in transitioners' aggressive behavior over time, and a slight decrease in such behavior for non-transitioners, $F(1,254)=$ $5.09, p=.02$.

\section{Discussion}

The majority of research on early sexual initiation during adolescence has predominantly focused on predictors of early initiation, in U.S. samples. The present study extended existing research by examining the complex relationship between early sexual initiation and a number of psychosocial factors (i.e., attachment, self-concepts, externalizing problems, and internalizing problems) among a sample of Dutch adolescents. Consistent with much of the research on early sexual initiation, the present study examined the predictive utility of the aforementioned psychosocial factors to early sexual initiation. However, the present work extended research in this area by 
Table 2 Mean scores (and SDs) for non-transitioners and transitioners males and females over the one-year period

\begin{tabular}{|c|c|c|c|c|c|c|c|c|c|c|c|c|}
\hline \multirow[t]{3}{*}{ Psychosocial variables } & \multicolumn{6}{|c|}{ Males (non-transitioner $n=186$; transitioner $n=26$ ) } & \multicolumn{6}{|c|}{ Females (non-transitioner $n=219 ;$ transitioner $n=39$ ) } \\
\hline & \multicolumn{2}{|c|}{ Time 1} & \multicolumn{2}{|c|}{ Time 2} & \multicolumn{2}{|c|}{ Across time $^{\mathrm{a}}$} & \multicolumn{2}{|c|}{ Time 1} & \multicolumn{2}{|c|}{ Time 2} & \multicolumn{2}{|c|}{ Across time $^{\mathrm{a}}$} \\
\hline & $M$ & $S D$ & $M$ & $S D$ & $M$ & $S D$ & $M$ & $S D$ & $M$ & $S D$ & $M$ & $S D$ \\
\hline \multicolumn{13}{|l|}{ Overall attachment mother ${ }^{\mathrm{b}}$} \\
\hline Non-transitioners & 4.80 & 1.16 & 4.59 & 1.16 & 4.70 & $1.16^{*}$ & 5.00 & 1.10 & 4.79 & 1.31 & 4.89 & $1.21 *$ \\
\hline Transitioners & 4.19 & 1.57 & 4.17 & 1.43 & 4.18 & $1.49 *$ & 4.58 & 1.07 & 4.55 & 1.05 & 4.56 & $1.05 *$ \\
\hline Across transition status ${ }^{\mathrm{c}}$ & 4.73 & 1.23 & 4.54 & 1.20 & & & 4.93 & 1.11 & 4.75 & 1.27 & & \\
\hline \multicolumn{13}{|l|}{ Overall attachment father ${ }^{\mathrm{b}}$} \\
\hline Non-transitioners & 4.52 & 1.24 & 4.39 & 1.37 & 4.45 & $1.31 *$ & 4.37 & 1.24 & 4.09 & 1.56 & 4.23 & 1.42 \\
\hline Transitioners & 3.99 & 1.25 & 3.98 & .97 & 3.98 & $1.11^{*}$ & 4.13 & 1.26 & 3.85 & 1.44 & 3.99 & 1.35 \\
\hline Across transition status ${ }^{\mathrm{c}}$ & 4.46 & 1.25 & 4.34 & 1.34 & & & 4.33 & 1.24 & 4.05 & 1.54 & & \\
\hline \multicolumn{13}{|l|}{ Overall attachment peers ${ }^{\mathrm{b}}$} \\
\hline Non-transitioners & 3.67 & 1.18 & 3.93 & 1.16 & 3.80 & 1.17 & 4.45 & 1.25 & 4.61 & 1.36 & 4.53 & 1.31 \\
\hline Transitioners & 3.59 & 1.18 & 4.36 & 1.06 & 3.97 & 1.18 & 4.34 & 1.13 & 4.35 & 1.33 & 4.34 & 1.23 \\
\hline Across transition status ${ }^{c}$ & 3.66 & $1.17 *$ & 3.98 & $1.15^{*}$ & & & 4.43 & 1.23 & 4.57 & 1.36 & & \\
\hline \multicolumn{13}{|l|}{ Scholastic competence ${ }^{\mathrm{b}}$} \\
\hline Non-transitioners & 2.29 & .32 & 2.31 & .33 & 2.30 & .33 & 2.36 & .33 & 2.38 & .34 & 2.37 & $.33 *$ \\
\hline Transitioners & 2.36 & .30 & 2.38 & .39 & 2.37 & .34 & 2.37 & .26 & 2.60 & .36 & 2.48 & $.33^{*}$ \\
\hline Across transition status ${ }^{c}$ & 2.30 & .32 & 2.32 & .34 & & & 2.36 & $.32 *$ & 2.41 & $.35^{*}$ & & \\
\hline \multicolumn{13}{|l|}{ Social acceptance ${ }^{\mathrm{b}}$} \\
\hline Non-transitioners & 2.44 & .27 & 2.43 & .30 & 2.43 & .28 & 2.46 & .29 & 2.46 & .27 & 2.46 & .28 \\
\hline Transitioners & 2.40 & .28 & 2.41 & .31 & 2.41 & .29 & 2.41 & .25 & 2.46 & .34 & 2.43 & .30 \\
\hline Across transition status ${ }^{\mathrm{c}}$ & 2.43 & .27 & 2.43 & .30 & & & 2.45 & .29 & 2.46 & .28 & & \\
\hline \multicolumn{13}{|l|}{ Athletic competence ${ }^{\mathrm{b}}$} \\
\hline Non-transitioners & 2.39 & .25 & 2.38 & .27 & 2.38 & .26 & 2.53 & .25 & 2.51 & .28 & 2.20 & .26 \\
\hline Transitioners & 2.39 & .26 & 2.38 & .35 & 2.38 & .30 & 2.51 & .28 & 2.41 & .36 & 2.46 & .32 \\
\hline Across transition status ${ }^{\mathrm{c}}$ & 2.39 & .25 & 2.38 & .28 & & & 2.53 & .26 & 2.50 & .29 & & \\
\hline \multicolumn{13}{|l|}{ Physical appearance ${ }^{\mathrm{b}}$} \\
\hline Non-transitioners & 2.44 & .31 & 2.45 & .26 & 2.45 & .28 & 2.47 & .26 & 2.45 & .26 & 2.46 & .26 \\
\hline Transitioners & 2.41 & .25 & 2.43 & .25 & 2.42 & .25 & 2.47 & .22 & 2.42 & .32 & 2.44 & .27 \\
\hline Across transition status ${ }^{c}$ & 2.44 & .30 & 2.45 & .26 & & & 2.47 & .25 & 2.44 & .27 & & \\
\hline \multicolumn{13}{|l|}{ Romantic appeal $^{\mathrm{b}}$} \\
\hline Non-transitioners & 2.48 & .28 & 2.50 & .27 & 2.49 & .27 & 2.56 & .29 & 2.59 & .29 & 2.57 & .29 \\
\hline Transitioners & 2.57 & .25 & 2.55 & .33 & 2.56 & .29 & 2.63 & .36 & 2.66 & .42 & 2.64 & .39 \\
\hline Across transition status ${ }^{\mathrm{c}}$ & 2.49 & .28 & 2.51 & .28 & & & 2.57 & .30 & 2.59 & .31 & & \\
\hline \multicolumn{13}{|l|}{ Close friendship ${ }^{\mathrm{b}}$} \\
\hline Non-transitioners & 2.44 & .37 & 2.43 & .33 & 2.44 & .35 & 2.47 & .38 & 2.49 & .36 & 2.48 & .37 \\
\hline Transitioners & 2.48 & .35 & 2.52 & .42 & 2.50 & .39 & 2.49 & .33 & 2.42 & .29 & 2.45 & .31 \\
\hline Across transition status ${ }^{c}$ & 2.45 & .37 & 2.44 & .34 & & & 2.48 & .37 & 2.47 & .35 & & \\
\hline Global self-worth $^{\mathrm{b}}$ & & & & & & & & & & & & \\
\hline Non-transitioners & 2.51 & .28 & 2.56 & .29 & 2.54 & .29 & 2.53 & .29 & 2.51 & .29 & 2.52 & .29 \\
\hline Transitioners & 2.55 & .32 & 2.60 & .34 & 2.57 & .33 & 2.44 & .30 & 2.47 & .31 & 2.47 & .31 \\
\hline Across transition status ${ }^{c}$ & 2.52 & .29 & 2.57 & .30 & & & 2.52 & .29 & 2.51 & .29 & & \\
\hline Rule-breaking behavior ${ }^{\mathrm{d}}$ & & & & & & & & & & & & \\
\hline Non-transitioners & .30 & .19 & .31 & .20 & .31 & $.19 *$ & .26 & .19 & .31 & .23 & .28 & $.21 *$ \\
\hline Transitioners & .46 & .23 & .48 & .28 & .47 & $.25^{*}$ & .37 & .19 & .50 & .28 & .43 & $.25^{*}$ \\
\hline Across transition status $^{c}$ & .32 & .20 & .33 & .21 & & & .28 & $.19 *$ & .34 & $.24 *$ & & \\
\hline Aggressive behavior $^{\mathrm{d}}$ & & & & & & & & & & & & \\
\hline Non-transitioners & .38 & .25 & .34 & .22 & .36 & $.23 *$ & .39 & .22 & .36 & .25 & .37 & $.24 *$ \\
\hline
\end{tabular}


Table 2 continued

\begin{tabular}{|c|c|c|c|c|c|c|c|c|c|c|c|c|}
\hline \multirow[t]{3}{*}{ Psychosocial variables } & \multicolumn{6}{|c|}{ Males (non-transitioner $n=186$; transitioner $n=26$ ) } & \multicolumn{6}{|c|}{ Females (non-transitioner $n=219 ;$ transitioner $n=39$ ) } \\
\hline & \multicolumn{2}{|c|}{ Time 1} & \multicolumn{2}{|c|}{ Time 2} & \multicolumn{2}{|c|}{ Across time $^{\mathrm{a}}$} & \multicolumn{2}{|c|}{ Time 1} & \multicolumn{2}{|c|}{ Time 2} & \multicolumn{2}{|c|}{ Across time $^{\mathrm{a}}$} \\
\hline & $M$ & $S D$ & $M$ & $S D$ & $M$ & $S D$ & $M$ & $S D$ & $M$ & $S D$ & $M$ & $S D$ \\
\hline Transitioners & .56 & .29 & .45 & .27 & .50 & $.28^{*}$ & .44 & .21 & .51 & .31 & .48 & $.27 *$ \\
\hline Across transition status ${ }^{\mathrm{c}}$ & .40 & $.26^{*}$ & .36 & $.23^{*}$ & & & .39 & .22 & .39 & .26 & & \\
\hline \multicolumn{13}{|l|}{ School problems ${ }^{\mathrm{d}}$} \\
\hline Non-transitioners & .29 & .21 & .33 & .23 & .31 & $.22 *$ & .33 & .19 & .37 & .27 & .35 & $.23 *$ \\
\hline Transitioners & .41 & .20 & .51 & .38 & .46 & $.31 *$ & .40 & .19 & .54 & .24 & .47 & $.23 *$ \\
\hline Across transition status ${ }^{c}$ & .30 & $.21 *$ & .35 & $.26^{*}$ & & & .34 & $.19 *$ & .40 & $.27 *$ & & \\
\hline \multicolumn{13}{|l|}{ Disobedience to parents ${ }^{\mathrm{d}}$} \\
\hline Non-transitioners & .31 & .28 & .35 & .28 & .33 & $.28^{*}$ & .34 & .27 & .35 & .30 & .34 & $.28 *$ \\
\hline Transitioners & .45 & .37 & .47 & .28 & .46 & $.32 *$ & .38 & .24 & .53 & .32 & .45 & $.29 *$ \\
\hline Across transition status ${ }^{c}$ & .32 & .29 & .36 & .28 & & & .34 & $.26^{*}$ & .37 & $.31 *$ & & \\
\hline \multicolumn{13}{|l|}{ Anxiety/depression ${ }^{\mathrm{d}}$} \\
\hline Non-transitioners & .26 & .22 & .22 & .21 & .24 & .22 & .37 & .29 & .35 & .30 & .36 & $.30 *$ \\
\hline Transitioners & .24 & .20 & .17 & .17 & .21 & .19 & .43 & .34 & .50 & .39 & .46 & $.37 *$ \\
\hline Across transition status ${ }^{\mathrm{c}}$ & .26 & .22 & .22 & .21 & & & .38 & .30 & .37 & .32 & & \\
\hline \multicolumn{13}{|l|}{ Withdrawal $^{\mathrm{d}}$} \\
\hline Non-transitioners & .32 & .28 & .32 & .31 & .32 & $.29 *$ & .39 & .31 & .39 & .30 & .39 & $.31 *$ \\
\hline Transitioners & .25 & .29 & .18 & .27 & .22 & $.28^{*}$ & .47 & .37 & .50 & .38 & .49 & $.37 *$ \\
\hline Across transition status ${ }^{c}$ & .31 & .28 & .31 & .30 & & & .40 & .32 & .40 & .32 & & \\
\hline \multicolumn{13}{|l|}{ Somatic complaints ${ }^{\mathrm{d}}$} \\
\hline Non-transitioners & .35 & .25 & .28 & .26 & .32 & .25 & .54 & .33 & .53 & .34 & .54 & $.34 *$ \\
\hline Transitioners & .35 & .26 & .29 & .24 & .32 & .24 & .70 & .39 & .70 & .40 & .70 & $.39 *$ \\
\hline Across transition status ${ }^{c}$ & .36 & .25 & .29 & .26 & & & .57 & .34 & .56 & .36 & & \\
\hline
\end{tabular}

* Significant main effect, $p<.05$

${ }^{a}$ Mean comparisons illustrating the main effect of transition status in the MANCOVA

b Absolute range $=1-4$

c Mean comparisons illustrating the main effect of time in the MANCOVA

d Absolute range $=0-2$

examining the relationship between early coitus and subsequent psychosocial development.

In terms of predictors of early sexual initiation, results partially supported our hypotheses for male adolescents. Low levels of maternal attachment and high levels of rule-breaking and aggression were associated with an increased likelihood of being an early initiator among our sample of male adolescents. However, self-concepts and internalizing problems did not predict to early initiation for this sample. Findings regarding predictors of early sexual initiation for females were inconsistent with our expectations. Contrary to our expectation, the results suggested that attachment, self-concept, externalizing problems, and internalizing problems did not predict early sexual initiation for females after controlling for type of education and ethnicity. Findings regarding externalizing problems were contrary to previous research suggesting that adolescents who report more externalizing problems are more likely to initiate sexual intercourse early (Donenberg et al., 2003). It is unclear why the present results differed from previous work in the area. However, it is important to note that levels of externalizing problems were low for the entire sample.

Regarding potential differences between transitioners and non-transitioners, multivariate results suggested no differential changes in attachment, self-concept, and internalizing and externalizing problems from Wave 1 to Wave 2 for transitioners and non-transitioners of either gender. However, the more sensitive assessment of difference by univariate repeated measures analyses suggested differential changes in some psychosocial factors for non-transitioner and transitioner female adolescents. Compared to female non-transitioners, female transitioners reported higher increases over time in disobedience to parents, school problems, and aggressive behavior as indicated by the significant time by initiation status interactions in the repeated measures analyses. These findings were 
consistent with research supporting early sexual initiation being a part of a problem behavior syndrome (Jessor \& Jessor, 1977). However, it is important to note that although transitioner females reported higher increases in problem behaviors over time, scale scores were low and did not indicate clinically significant levels of problem behaviors for any group.

While the findings illustrate associations between early sexual initiation and some problem behaviors for female adolescents, results did not support a negative relationship between early coitus and self-perception. With the exception of perceptions of scholastic competence, there were no differential changes in self-perception between female transitioners and non-transitioners over the two time periods. Interestingly, the scholastic self-perceptions of early female transitioners increased in the period following transition, whereas no change was found for the non-transitioners. These results were contrary to previous studies that have found early sexual intercourse to be associated with low perceptions of school competence among female adolescents (Longmore et al., 2004). These differences may have to do with the different measures of perceptions of school competence used or broader differences in the culture of the populations studied.

Although data from this study did not lend itself to explanations of similarities or differences found between present findings and previous research, it is possible that the differences have to do, at least in part, with sociocultural differences between specific samples represented in research studiesparticularly that of the United States and the Netherlands. Various research has documented the divergent cultural views regarding adolescent sexuality in the United States and in the Netherlands (Ehrhardt, 1996; Dodge, Sandfort, Yarber, \& de Wit, 2005; Schalet, 2000, 2007). Whereas many have documented Americans' views of adolescent sexuality to entail visions of biologically driven urges that interfere with rational thought (Ehrhardt, 1996; Schalet, 2000), the prominent Dutch view of adolescent sexual activity is one of normal adolescent development (Dodge et al., 2005; Schalet, 2000). This perspective is indicated in the "sex positive" approach of Dutch nationally-funded comprehensive sexual education programs (Ferguson, Vanwesenbeeck, \& Knijn, 2008; Weaver, Smith, \& Kippax, 2005). In the context of Dutch society, sexuality is generally not viewed as an experimental and risky individual activity, but part of forming responsible and respectful intimate relationships (Schalet, 2000, 2007).

The divergent cultural perspectives on the meaning of adolescent sexuality between American and Dutch societies may, in fact, be associated with different associations between early sexual initiation and psychosocial problems for adolescents. In a culture where many believe adolescents to be capable of exercising control over their sexuality, sexual activity during adolescence is a developmental task - a sign of independence and autonomy, rather than a form of deviancy. In such contexts, the act of an adolescent engaging in sexual activity at earlier ages may signify precocious development in terms of starting the transition to adulthood earlier, rather than a problem behavior. This view of adolescent sexuality may explain the relatively low levels of externalizing and internalizing problems among transitioners in this sample, as well as the higher perceptions of academic competence among transitioner females.

Results must be interpreted in the context of study limitations. First, we used an existing dataset designed to explore problem behaviors in general, and not experiences of sexual initiation. Therefore, the item used to measure participants' sexual history did not identify type of sexual activity. Sexual activity was measured in the study with an item that used the broad term of having "slept with somebody." Although the phrase is commonly understood in the Netherlands to mean penile-vaginal intercourse, there may be adolescents for whom this term may hold different meanings (e.g., oral sex, mutual masturbation, sleeping, etc.).

The second limitation of the study is related to the first. There are various other factors of sexual experience that may be related to psychosocial consequences (e.g., having a positive or negative first experience) that could not be explored with the data available. By measuring sexual experience with only one item, the present study provides limited information into the nature and experience of early initiation for adolescents. We therefore cannot explore how various factors could mediate or moderate the relationship between sexual intercourse and the psychosocial factors examined. Third, the study relied on participant self-report that may be subject to various biases, including social desirability and reporter bias. However, this is a problem associated with all research utilizing self-report methods (O'Sullivan, 2008).

The present study suggests that while some of the associations found in previous research between early sexual initiation and various problem behaviors held true for the adolescent females in this Dutch sample, there were also important differences. For one, both transitioners and nontransitioners had very low levels of problem behaviors. These levels were, in fact, too low to be considered part of a problem behavior syndrome as suggested in previous research. Second, transitioner females reported higher levels of academic self-concept than their non-transitioner peers. Although not directly tested in this study, possible reasons for such differences may be due to the meaning of adolescent sexuality within Dutch culture. In a society where adolescents are taught and expected to participate in responsible sexual relationships, sexual activity during adolescence may be a healthy part of adolescent development (Schalet, 2000, 2007).

Findings from this study suggest that early sexual initiation may not be inherently problematic. These findings are important because it points toward the possibility of potential protective factors that may serve to promote sexual health for adolescents who engage in sexual initiation prior to their peers. Future developmental research should examine macrosystemic 
factors, including specific aspects of culture and social context (e.g., societal beliefs, values, practices), that place adolescents at risk for, and protect against the negative outcomes commonly associated with early coitus. Furthermore, future research should examine the nature and experience of early initiation for adolescents, and how these experiences relate to subsequent development.

Acknowledgement Data analysis and manuscript writing was supported by a grant from the National Institute of Mental Health to the HIV Center for Clinical and Behavioral Studies (P30-MH43520; PI: Anke E. Ehrhardt, Ph.D.).

Open Access This article is distributed under the terms of the Creative Commons Attribution Noncommercial License which permits any noncommercial use, distribution, and reproduction in any medium, provided the original author(s) and source are credited.

\section{References}

Achenbach, T. (1991). Manual for the child behavior checklist/4-18 and 1991 profile. Burlington, VT: University of Vermont, Department of Psychiatry.

Alloy, L., Zhu, L., \& Abramson, L. (2003). Cognitive vulnerability to depression: Implications for adolescent risk behavior in general. In D. Romer (Ed.), Reducing adolescent risk: Toward an integrated approach (pp. 171-182). Thousand Oaks, CA: Sage.

Arbona, C., \& Power, T. G. (2003). Parental attachment, self-esteem, and antisocial behaviors among African American, European American, and Mexican American adolescents. Journal of Counseling Psychology, 50, 40-51.

Armour, S., \& Haynie, D. L. (2007). Adolescent sexual debut and later delinquency. Journal of Youth and Adolescence, 36, 141-152.

Armsden, G., \& Greenberg, M. (1987). The inventory of parent and peer attachment: Individual differences and their relationship to psychological well-being in adolescence. Journal of Youth and Adolescence, 16, 427-453.

Biglan, A., Metzer, C., Wirt, R., Ary, D., Noell, J., Ochs, L., ... Hood, D. (1990). Social and behavioral factors associated with high-risk sexual behavior among adolescents. Journal of Behavioral Medicine, 15, 245-261.

Black, M., Ricardo, I., \& Stanton, B. (1997). Social and psychological factors associated with AIDS risk behaviors among low income, urban, African-American adolescents. Journal of Research on Adolescence, 7, 173-195.

Brack, C. J., Brack, G., \& Orr, D. P. (1994). Dimensions underlying problem behaviors, emotions, and related psychosocial factors in early and middle adolescents. Journal of Early Adolescence, 14, 345-370.

Bronfenbrenner, U. (1979). The ecology of human development: Experiments by nature and design. Cambridge: Harvard University Press.

Caminis, A. P., Henrich, C. H., Ruchkin, V., Schwab-Stone, M. E., \& Martin, A. (2007). Psychosocial predictors of sexual initiation and high-risk sexual behaviors in early adolescence. Child and Adolescent Psychiatry and Mental Health, 1, 1-14.

Cavazos-Rehg, P. A., Spitznagel, E. L., Bucholz, K. K., Nurnberger, J., Edenberg, H. J., Kramer, J. R., ... Bierut, L. J. (2009). Predictors of sexual debut at age 16 or younger. Archives of Sexual Behavior. doi:10.1007/s10508-008-9397-y.

Cooper, M. L., Shaver, P. R., \& Collins, N. L. (1998). Attachment styles, emotion regulation, and adjustment in adolescence. Journal of Personality and Social Psychology, 74, 1380-1397.
Crockett, L. J., Moilanen, K. L., Raffaelli, M., \& Randall, B. A. (2006). Psychological profiles and adolescent adjustment: A person-centered approach. Development and Psychopathology, 18, 195-214.

Davis, E. C., \& Friel, L. V. (2001). Adolescent sexuality: Disentangling the effects of family structure and family context. Journal of Marriage and the Family, 63, 669-681.

de Graaf, H., Vanwesenbeeck, I., Meijer, S., Woertman, L., \& Meeus, W. (2009). Sexual trajectories during adolescence: Relation to demographic characteristics and sexual risk. Archives of Sexual Behavior, 38, 276-282.

Dekovic, M. (1999). Risk and protective factors in the development of problem behavior during adolescence. Journal of Youth and Adolescence, 28, 667-685.

Dekovic, M., Pels, T., \& Model, S. (2006). Child rearing in six ethnic families: The multi-cultural Dutch experience. Lewiston: Edwin Mellen Press.

Dodge, B., Sandfort, T. G. M., Yarber, W., \& de Wit, J. (2005). Sexual health among male college students in the United States and The Netherlands. Journal of Health Behavior, 29, 172-182.

Donenberg, G. R., Bryant, F. B., Emerson, E., Wilson, H. W., \& Pasch, K. E. (2003). Tracing the roots of early sexual debut among adolescents in psychiatric care. Journal of the American Academy of Child and Adolescent Psychiatry, 42, 594-608.

Ehrhardt, A. A. (1996). Our view of adolescent sexuality: A focus on risk behavior without the developmental context. American Journal of Public Health, 86, 1523-1525.

Ferguson, R. M., Vanwesenbeeck, I., \& Knijn, T. (2008). A matter of facts... and more: An exploratory analysis of the content of sexuality education in The Netherlands. Sex Education, 8, 93-106.

Flannery, D., Vazsonyi, A. T., \& Rowe, D. C. (1996). Caucasian and Hispanic early adolescent substance use: Parenting, personality, and school adjustment. Journal of Early Adolescence, 16, 71-89.

Fletcher, A., Steinberg, L., \& Sellers, E. (1999). Adolescents' wellbeing as a function of perceived interparental consistency. Journal of Marriage and the Family, 61, 599-610.

Gillmore, M., Hawkins, J., Catalano, R., Day, L., Moore, M., \& Abbott, R. (1991). Structure of problem behaviors in preadolescence. Journal of Consulting and Clinical Psychology, 59, 499-506.

Greenberg, J., Magder, L., \& Aral, S. (1992). Age at first coitus: A marker of risky sexual behavior in women. Sexually Transmitted Diseases, 19, 331-334.

Harter, S. (1988). Manual for the Self-Perception Profile for Adolescents. Denver: University of Denver.

Houlihan, A. E., Gibbons, F. X., Gerrard, M., Yeh, H., Reimer, R. A., \& Murry, V. M. (2008). Sex and the self: The impact of early sexual onset on the self-concept and subsequent risky behavior of African American adolescents. Journal of Early Adolescence, 28, 70-91.

Jessor, R., \& Jessor, S. L. (1977). Problem behavior and psychosocial development: A longitudinal study of youth. New York: Academic Press.

Ketterlinus, R., Lamb, M., \& Nitz, K. (1994). Adolescent nonsexual and sex-related problem behaviors: Their prevalence, consequences, an co-occurrence. In R. Ketterlinus \& M. E. Lamb (Eds.), Adolescent problem behaviors, issues, and research (pp. 17-39). Hillsdale, NJ: Erlbaum.

Kirby, D., Lepore, G., \& Ryan, J. (2005). Factors affecting teen sexual behavior, pregnancy, childbearing and sexually transmitted disease: Which are important? Which can you change? Washington, DC: The National Campaign to Prevent Teen Pregnancy.

Krieger, N. (2001). Theories for social epidemiology in the 21 st century: An ecosocial perspective. International Journal of Epidemiology, 30, 668-677.

Laflin, M. T., Wang, J., \& Barry, M. (2008). A longitudinal study of adolescent transition from virgin to nonvirgin status. Journal of Adolescent Health, 42, 228-236. 
Lerner, R., \& Galambos, N. (1998). Adolescent development: Challenges and opportunities for research, programs, and policy. Annual Review of Psychology, 49, 413-446.

Lieberman, M., Doyle, A., \& Markiewicz, D. (1999). Developmental patterns in security of attachment to mother and father in late childhood and early adolescence: Associations with peer relations. Child Development, 70, 202-213.

Liu, Y. (2006). Paternal/maternal attachment, peer support, social expectations of peer interaction, and depressive symptoms. Adolescence, $41,705-721$.

Longmore, M. A., Manning, W. D., Giordano, P. C., \& Rudolf, J. L. (2004). Self-esteem, depressive symptoms, and adolescents' sexual onset. Social Psychology Quarterly, 67, 279-295.

Maggs, J., Almeida, D., \& Galambos, N. (1995). Risky business: The paradoxical meaning of problem behavior for young adolescents. Journal of Early Adolescence, 15, 344-362.

Manning, W. D., Longmore, M. A., \& Giordano, P. G. (2005). Adolescents' involvement in non-romantic sexual activity. Social Science Research, 34, 384-407.

Martin, K. (1996). Puberty, sexuality and the self: Girls and boys at adolescence. New York: Routledge.

Masten, A. S., Coatsworth, J. D., Neemann, J., Gest, S. D., Tellegen, A., \& Garmezy, N. (1995). The structure and coherence of competence from childhood through adolescence. Child Development, 66, 1635-1659.

Miller, B., Norton, M., Curtis, T., Hill, E., Schvaneveldt, P., \& Young, M. (1997). The timing of sexual intercourse among adolescents: Family, peer and other antecedents. Youth and Society, 29, 54-83.

O'Sullivan, L. F. (2008). Challenging our assumptions regarding the validity of self-report measures: The special case of sexual behavior [Editorial]. Journal of Adolescent Health, 42, 207-208.

Paul, C., Fitzjohn, J., Herbison, P., \& Dickson, N. (2000). The determinants of sexual intercourse before age 16. Journal of Adolescent Health, 27, 136-147.

Peeters, J. (1994). Klachtenlijst voor adolescenten: KLAD [Complaintlist for adolescents]. Gedragstherapie, 27, 109-125.

Raja, S., McGee, R., \& Stanton, W. (1992). Perceived attachments to parents and peers and psychological well-being in adolescence. Journal of Youth and Adolescence, 21, 471-485.

Ravesloot, J. (1997). Seksualiteit in de Jeugdfase Vroeger en Nu: Ouders en Jongeren aan het Woord. Amsterdam: Het Spinhuis.

Ream, G. (2006). Reciprocal effects between the perceived environment and heterosexual intercourse among adolescents. Journal of Youth and Adolescence, 35, 771-785.

Reitz, E., Dekovic, M., \& Meijer, A. (2005). The structure and stability of externalizing and internalizing problem behavior during early adolescence. Journal of Youth and Adolescence, 34, 577-588.

Reitz, E., Dekovic, M., \& Meijer, A. (2006). Longitudinal relations among parenting, best friends, and early adolescent problem behavior: Testing bidirectional effects. Journal of Early Adolescence, 26, 272295.

Rogoff, B. (2003). The cultural nature of human development. New York: Oxford University Press.

Rothbaum, F., \& Weisz, J. (1994). Parental caregiving and child externalizing behavior in nonclinical samples: A meta-analysis. Psychological Bulletin, 116, 55-74.

Salazar, L. F., Crosby, R. A., DiClemente, R. J., Wingood, G. M., Lescano, C. M., Brown, L. K., ... Davies, S. (2005). Self-esteem and theoretical mediators of safer sex among African American female adolescents: Implications for sexual risk reduction interventions. Health Education and Behavior, 32, 413-427.

Salazar, L. F., DiClemente, R. J., Wingood, G. M., Crosby, R. A., Harrington, K., Davies, S., ... Oh, M. K. (2004). Self-concept and adolescents' refusal of unprotected sex: A test of mediating mechanisms among African American girls. Prevention Science, 5, 137 149
Sandfort, T. G. M., Orr, M., Hirsch, J. S., \& Santelli, J. (2008). Long-term health correlates of timing of sexual debut: Results from a national US study. American Journal of Public Health, 98, 155-161.

Santelli, J. S., Kaiser, J., Hirsch, L., Radosh, A., Simkin, L., \& Middlestadt, S. (2004). Initiation of sexual intercourse among middle school adolescents: The influence of psychosocial factors. Journal of Adolescent Health, 34, 200-208.

Savin-Williams, R., \& Diamond, L. M. (2004). Sex. In R. Lerner \& L. Steinberg (Eds.), Handbook of adolescent psychology (pp. 189-231). Hoboken, NJ: Wiley.

Schalet, A. (2000). Raging hormones, regulated love: Adolescent sexuality and the constitution of the modern individual in the United States and the Netherlands. Body and Society, 6, 75-105.

Schalet, A. (2007). Adolescent sexuality viewed through two different cultural lenses. In T. Mitchell \& A. Owens (Eds.), Sexual health. Vol. 3: Moral and cultural foundations (pp. 365-387). Westport, CT: Praeger Publishers.

Small, S., \& Luster, T. (1994). Adolescent sexual activity: An ecological, risk factor approach. Journal of Marriage and Family, 56, 181-192.

Smetana, J. G., Campione-Barr, N., \& Metzger, A. (2006). Adolescent development in interpersonal and societal contexts. Annual Review of Psychology, 57, 255-284.

Smith, C. A. (1997). Factors associated with early sexual activity among urban adolescents. Social Work, 42, 334-346.

Sokol-Katz, J., Dunham, R., \& Zimmerman, R. (1997). Family structure versus parental attachment in controlling adolescent deviant behavior: A social control model. Adolescence, 32, 199-215.

Steinberg, L., \& Morris, A. S. (2001). Adolescent development. Annual Review of Psychology, 52, 83-110.

Stevens, J. (1992). Applied multivariate statistics for the social sciences. Hillsdale, NJ: Lawrence Erlbaum Associates.

Stevens, J. (2001). The social ecology of the co-occurrence of substance use and early coitus among poor, urban black female adolescents. Substance Use and Misuse, 36, 421-446.

Swertz, O., Duimelaar, P., Thijssen, J., Harmsen, C., \& Vergeer, J. (2003). Allochtonen in Nederland 2003 [Immigrants in the Netherlands 2003]. Voorburg/Heerlen: Centraal Bureau voor de Statistiek.

Verhulst, F. C., Van der Ende, J., \& Koot, H. M. (1997). Handleiding voor de Youth Self Report (YSR) [Guide for the Youth Self Report]. Rotterdam, The Netherlands: Erasmus University, Department of Child and Adolescent Psychiatry.

Vermeiren, R., Bogaerts, J., Ruchkin, V., Deboutte, D., \& SchwabStone, M. (2004). Subtypes of self-esteem and self-concept in adolescent violent and property offenders. Journal of Child Psychology and Psychiatry, 45, 405-411.

Weaver, H., Smith, G., \& Kippax, S. (2005). School-based sex education policies and indicators of sexual health among young people. Sex Education, 5, 171-188.

Webb, J., Baer, P., McLaughlin, R., McKelvey, R., \& Caid, C. (1991). Risk factors and their relation to initiation of alcohol use among early adolescents. Journal of the American Academy of Child and Adolescent Psychiatry, 30, 563-568.

Williams, T., Connolly, J., \& Cribbie, R. (2008). Light and heavy heterosexual activities of young Canadian adolescents: Normative patterns and differential predictors. Journal of Research on adolescence, 18, 145-172.

Williams, S. K., \& Kelly, F. D. (2005). Relationships among involvement, attachment, and behavioral problems in adolescence: Examining father's influence. Journal of Early Adolescence, 25, 168-196.

Winters, K. C., August, G., \& Leitten, W. (2003). Preventive interventions for externalizing disorders in adolescents. In D. Romer (Ed.), Reducing adolescent risk: Toward an integrated approach (pp. 139-146). Thousand Oaks, CA: Sage. 
Woodward, L., Fergusson, D., \& Horwood, J. (2001). Risk factors and life processes associated with teen pregnancy: Results of a prospective study from birth to 20 years. Journal of Marriage and Family, 63, 1170-1184.
Worthington, R. L., Savoy, H., Dillon, F. R., \& Vernaglia, E. R. (2002). Heterosexual identity development: A multidimensional model of individual and social identity. The Counseling Psychologist, 30, 496-531. 